\title{
Numerical Investigation and Design of Optical on Chip Waveguide With Engineered Dispersion for Generation of Supercontinuum Based Frequency Combs
}

\author{
Mohammad Reza Alizadeh \\ Shahid Rajaee Teacher Training University \\ Saeed Olyaee \\ Shahid Rajaee Teacher Training University \\ Mahmood Seifouri ( $\square$ mahmood.seifouri@sru.ac.ir) \\ Shahid Rajaee Teacher Training University
}

\section{Research Article}

Keywords: supercontinuum generation, frequency comb generation, dispersion, zero-dispersion wavelength, highly nonlinear

Posted Date: February 7th, 2022

DOI: https://doi.org/10.21203/rs.3.rs-1050436/v1

License: (c) (1) This work is licensed under a Creative Commons Attribution 4.0 International License.

Read Full License 


\title{
Numerical investigation and design of optical on chip waveguide with engineered dispersion for generation of supercontinuum based frequency combs
}

\author{
Mohammad Reza Alizadeh ${ }^{1}$, Saeed Olyaee ${ }^{2}$, Mahmood Seifouri*,1 $^{*}$ \\ ${ }^{1}$ Faculty of Electrical Engineering, Shahid RajaeeTeacher Training University, Tehran, Iran \\ ${ }^{2}$ Nano-Photonics and Optoelectronics Research Laboratory (NORLab), Shahid Rajaee Teacher Training \\ University, Tehran, Iran
}

\begin{abstract}
In this paper, with dispersion engineering, two waveguides with silicon core and $\mathrm{SiO}_{2}$ cladding are proposed to generation the supercontinuum spectrum and optical frequency combs. By injecting a pulse with a peak power of 800 (W) and a pulse duration of 50 (fs), the output supercontinuum spectrum is obtained from a wavelength of $1100(\mathrm{~nm})$ to $4000(\mathrm{~nm})$. Also, by applying a maximum power of 1 KW and a pulse width of 100 (fs), optical frequency combs based on a supercontinuum with a broadband frequency combs have been obtained. Our proposed structure has good potential for engineering and achieving flatness dispersion which there are two zero dispersion wavelengths at 1890 (nm) and 2850 $(\mathrm{nm})$. This flat dispersion can be very useful to achieve the desired output spectrum. Due to the materials used and the flat structure of the proposed waveguides, these waveguides can be used for integrated optical circuits as well as applications in optical communications, spectroscopy, and sensors.
\end{abstract}

Key words: supercontinuum generation, frequency comb generation, dispersion, zero-dispersion wavelength, highly nonlinear.

\footnotetext{
* Corresponding author. Email: Mahmood.Seifouri@sru.ac.ir
} 


\section{Introduction}

Today, the need to use high-capacity applications such as high- capacity data transmission such as high-resolution video, artificial intelligence, and 5G technologies has led to a high-capacity transmission network. Optical networks have enough potential to reply for these needs due to their large bandwidth. In addition, the capacity of optical networks is increasing rapidly. Due to the high bandwidth, optical signal processing (OSP) techniques are considered a suitable platform for processing high data capacities (Qin. Jun et al.2020; Dupont et al. 2012; Husakou. A. V. and J. Herrmann 2001). OSP systems are used in various optical fields such as analog and digital signal processing, nonlinear applications, and fast and advanced data modulation. They are essential for the processing of high-speed fiber-optic communication signals (Willner et al. 2019).

Most recently, there is urgent need for compression and device miniaturization in optical circuits, which can be achieved by using the nonlinear effects of structures containing silicon (Li et al. 2018), Hydex (Moss et al. 2013 ), SiN (Tan et al 2018; Frigg et al. 2019), AlN (Gong et al. 2018), diamond (Amirhassan et al. 1019), IIIV semiconductor ( $\mathrm{Pu}$ et al. 2016; $\mathrm{Hu}$ et al. 2018; Chang et al. 2018), and chalcogenide (Karami et al. 2017; Alizadeh and Seifouri 2017; Seifouri and Alizadeh 2018; Alizadeh and Seifouri 2019). Silicon-on-insulator (SOI) waveguide is considered as an important substrate in compact circuits due to its high nonlinear properties and compatibility with CMOS integrated circuits.

There are several ways to increase the nonlinear coefficient of the structure. One of the methods is a strong optical field confinement in the structure, which increases with a high index contrast between the core and the cladding, which results in a highly efficient four-wave mixing (FWM) process that is important in many applications ( $\mathrm{Pu}$ et al. 2017; Oxenlowe et al. 2012). The FWM phenomenon is 
especially important for phase-sensitive processing where signal and idler wave interference is desired ( $\mathrm{Da}$ et al. 2016). One of the most important applications of nonlinear effects is supercontinuum spectrum generation (SCG) as well as the optical frequency comb generation (FCG).

The supercontinuum is the broadening of a very short pulse that enters to input of a waveguide or fiber with a high nonlinear coefficient. The interaction of various parameters such as dispersion and nonlinear effects such as self-phase modulation, Raman effects, and four-wave mixing, etc., cause this expansion. Typically, the broad SCG is formed from compressing of solitons and soliton breaking effect in the anomalous dispersion region, and then the propagation of the dispersive wave (Dupont et al. 2012; Husakou and Herrmann 2001). SCG sources include wide and flat output spectra that have been used extensively in spectroscopy (Ke et al 2009; Eggleton 2011), microscopy (Aggarwal and Sanghera 2002), imaging (Dudley et al. 2006), metrology, tomography, pulse compression, fingerprinting, and sensors (Dudley and Taylor 2009; Paul et al. 2019). Especially the infrared spectral range is very important because the molecules of the most of materials oscillate in this region (Dai et al. 2018). The SCG has been extensively studied in nonlinear media such as fibers and photonic crystal fibers. However, the waveguides that can be implemented on chips have recently attracted much attention (Yoon et al. 2017; Safioui et al. 2014). In addition to optimizing the optical waveguide structure, the appropriate pumping wavelength and maximum input pulse power can be used to broaden the pulse. However, it should be noted that in practice, there are limitations for each. The dispersion profile also plays an important role in the width and flattening of the SCG, especially if the pumping wavelength is close to the zero dispersion wavelengths (ZDW), which can be adjusted based on the waveguide structure in urgent regime. To increase the width of the produced 
spectrum, pumping is injected to the anomalous dispersion zone, which produces solitons and four-wave mixing (Møller et al. 2015; Luo et al. 2017). The width of the supercontinuum spectrum in fibers is usually greater than in waveguides and fibers have lower losses. However, to produce a wide spectrum, the effective length of the waveguides is shorter than that of the fibers, and the flat nature of the waveguides allows them to be implemented on chips (Yu et al. 2014; McCarthy et al. 2013; Ma et al. 2013).

The optical frequency comb (OFC) consists of a spectrum with equal frequency lines, each of which has a relatively constant phase so that the spectrum appears as a comb. This OFC spectrum can be employed in many applications such as astronomy (Obrzud et al. 2019), optical communications (Feifle et al. 2014), microwave photonics (Xue et al. 2018), and other fields. Several methods have been proposed for creating frequency combs with a flat spectrum for use in highcapacity optical communications (Hu and Oxenløwe 2021). The first method is to use combs based on electro-optical modulators. However, this method usually involves bulky components, and has limited comb lines spanning over a few nanometers or exhibit the triangular spectrum (Company and Weiner 2014; Zhang et al. 2019). The second method is to use integrated mode-locked lasers. In this method, the optical linewidth of the mode-locked laser is usually too large for coherent optical communication (Zhang et al. 2016; Liu et al. 2020). The third method is to use the nonlinear microresonator-based Kerr frequency combs. In this method, the spacing of frequency comb depends on the free spectral range (FSR) of the used microresonator, where its tunability is limited (Kippenberg et al. 2018). The fourth method is based on SCG by injecting a laser pulse into a fiber or optical waveguide suitable for practical application because of the long time stability of the comb (Obrzud et al.2018; Deng et al. 2022). Highly nonlinear fiber with 
normal dispersion is a desirable method for generation of broadband OFC spectrum (Takushima et al.1998). However, such a fiber must be hundreds of meters long and should exhibit a normal, almost near zero-dispersion along this length (Myslivets et al.2012). The production of long fiber with the mentioned characteristics has serious challenges in manufacturing technology. One method is to use high nonlinear optical integrated circuits based waveguides witch not only using materials with high nonlinear coefficients but also the high optical limitation can be achieved in these waveguides; thus, the desired length in these structures is significantly reduced. In this paper, by dispersion engineering and investigating the effect of dimensions on the dispersion profile, two silicon waveguides are proposed to generation of supercontinuum spectrum and frequency combs based on SCG. Due to the materials used and the flat structure of the proposed waveguides, these waveguides can be used for integrated optical circuits as well as applications in optical communications, spectroscopy, and sensors.

\section{theory}

The nonlinear Schrödinger equation can be solved numerically using the split-step Fourier method to study the generation of supercontinuum spectrum and frequency combs. The simplified form of this equation is as follows (Alizadeh and Seifouri 2020):

$\frac{\partial A}{\partial Z}=(\widehat{D}+\widehat{N}) A$

Where $\mathrm{D}$ represents the linear part and $\mathrm{N}$ represents the nonlinear part of this equation. The value of $\mathrm{D}$ is obtained from the following equation.

$D=-\frac{\alpha(w)}{2}+\sum_{n \geq 2} \beta_{n} \frac{i^{n+1}}{n !} \frac{\partial^{n}}{\partial t^{n}}$ 
In equation $2, \alpha$, represents the waveguide loss coefficient, which can be neglected due to the low waveguide length. $\beta_{n}(n \geq 2)$ Indicates the various orders of dispersion that can be obtained from the following equation (Zho and Brown 2002; Ming et al. 2010).

$\beta_{m}=\left(\frac{d^{m} \beta}{d \omega^{m}}\right)_{\omega=\omega_{0}} \quad(m=0,1,2, \ldots)$

In this equation, $\omega_{0}$ represents the pumping wavelength. In addition, the nonlinear part of the equation 1 is obtained from the follows (Karpisz et al. 2015):

$$
N=i \gamma\left(1+\frac{i}{w_{0}} \frac{\partial}{\partial t}\right) \int_{-\infty}^{\infty} R\left(T^{\prime}\right)\left|A\left(Z, T-T^{\prime}\right)\right|^{2} d T^{\prime}
$$

In this equation, $\mathrm{A}$ is the input pump envelope, and $\gamma$ is the nonlinear coefficient and is obtained from the following equation:

$\gamma=\frac{\omega_{0} n_{2}}{c A_{\text {eff }}}=\frac{2 \pi n_{2}}{\lambda A_{\text {eff }}}$

In the above equation $n_{2}$, the nonlinear refractive index is related to the Kerr effect. $\mathrm{C}$ is the speed of light in a vacuum. $A_{\text {eff }}$ is the effective mode area at the central wavelength for the propagation mode and $\lambda$ is the pumping wavelength. Also, in Equation 4, $\mathrm{R}\left(T^{\prime}\right)$ is a function of the Raman response and is obtained from the following equation (Ghanbari et al. 2018).

$$
R\left(T^{\prime}\right)=\left(1-f_{R}\right) \delta(T)+f_{R} h_{R}(T)
$$

Where the value of $f_{R}=0.1$ and $h_{R}(\mathrm{~T})$ is obtained from the following equation:

$$
h_{R}(T)=\frac{\tau_{1}^{2}+\tau_{2}^{2}}{\tau_{1} \tau_{2}^{2}} \exp \left(-\frac{t}{\tau_{2}}\right) \sin \left(\frac{t}{\tau_{1}}\right)
$$


Where the value of $\tau_{1}=10 \mathrm{fs}$ and $\tau_{2}=3 \mathrm{ps}$ for $\mathrm{Si}$ (Ghanbari et al. 2018).

The group velocity dispersion $\mathrm{D}(\lambda)$ is calculated using the propagation mode wavelength-dependent effective refractive index through the following equation

$$
D(\lambda)=-\frac{\lambda}{c} \frac{\partial^{2} \operatorname{Re}\left(n_{e f f}\right)}{\partial \lambda^{2}}
$$

Where $\operatorname{Re}\left(n_{e f f}\right)$ is the real part of the effective refractive index and determined by using of the Sellmeier equation (Agrawal 2013).

\section{Dispersion engineering and waveguide design}

We have designed two waveguides with silicon core and $\mathrm{SiO}_{2}$ cladding to produce supercontinuum spectrum and frequency combs. To achieve the final structure, we have used the effect of waveguide dimensional changes on dispersion and dispersion engineering. Fig1 shows the structure of the two proposed waveguides. The first structure in Figure 1(a)-(b) is very familiar, while the second structure is suitable for flat dispersion applications (Guo et al. 2019).

Fig1(e) shows the dispersion profile of the first structure. In this structure, the height $\mathrm{h}$ adjust $300(\mathrm{~nm})$, and the width $\mathrm{w}$ is equal to $\mathrm{w}=1000(\mathrm{~nm})$. The curve has two zero-dispersion wavelengths around the wavelengths of 1980 (nm) and $2800(\mathrm{~nm})$. Fig2 shows the dispersion profile of structure 2, in which 2 (a), 2 (b), 2 (c), and 2 (d) correspond to the heights $h_{1}, h_{2}, h_{3}, h_{4}$, respectively, and Fig2(e) is related to width changes of the waveguide.

The selected dimensions for structure two are shown in Table 1. This structure has two zero-dispersion wavelengths in $1890(\mathrm{~nm})$ and $2850(\mathrm{~nm})$ in the selected dimensions. However, the maximum dispersion in its anomalous region is much 
less than in structure 1, which confirms the flatness of the dispersion curve in structure 2 compared to structure 1.

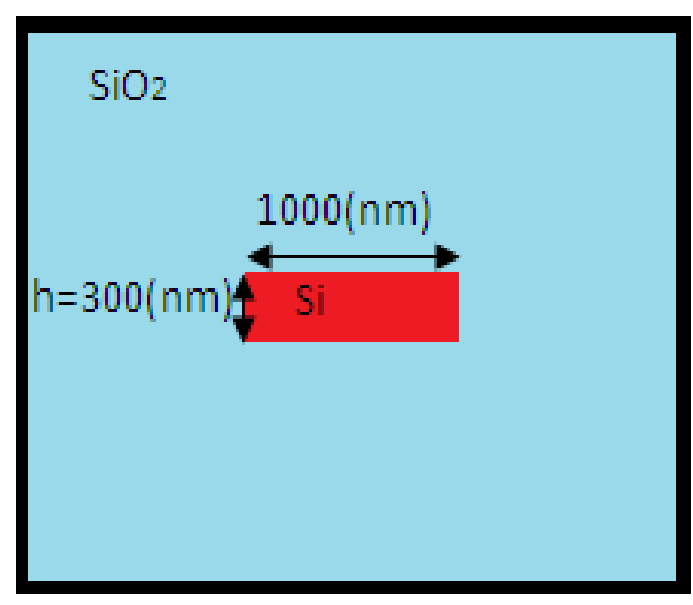

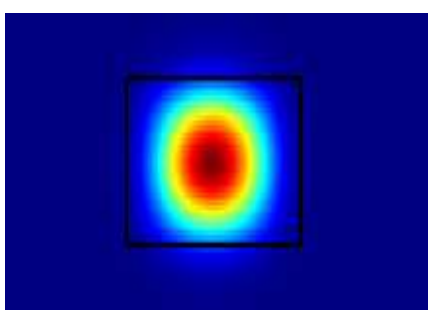

(b)

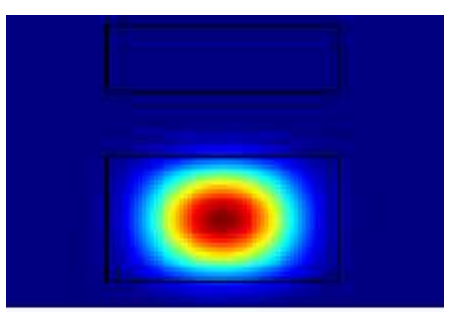

(d)

(a)

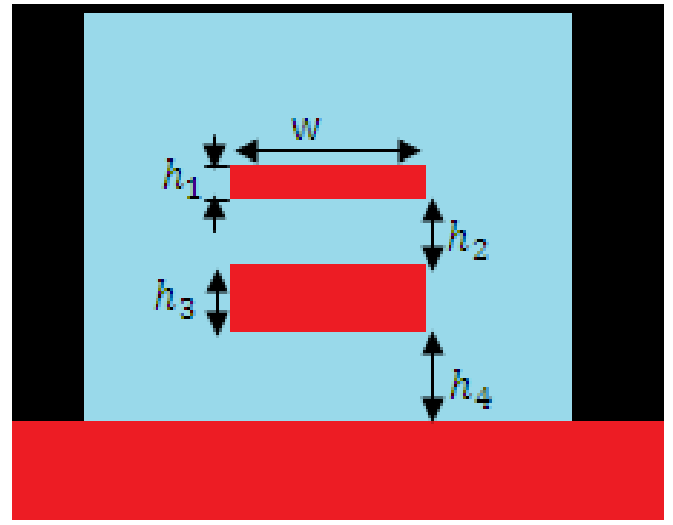

(c)

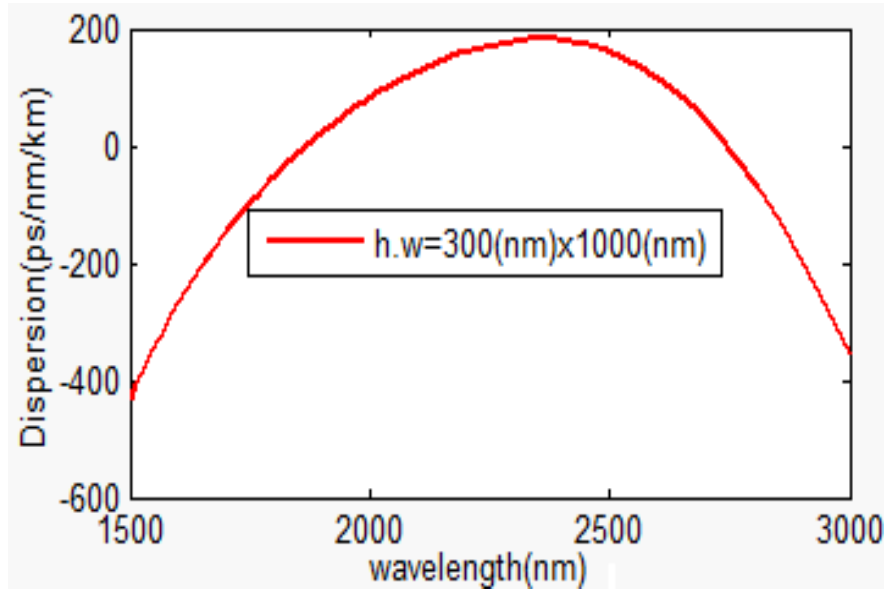

(e)

Fig1: The waveguides studied in this research (a) and (b) the shape and mode profile of the first structure, (c) and (d) the structure and mode profile of the second proposed structure and (e) Dispersion index of structure 1, in this structure, the size of height $\mathrm{h}$ is equal to $300(\mathrm{~nm})$, and the size of width $\mathrm{w}$ is equal to $\mathrm{w}=1000(\mathrm{~nm})$. The curve has two zero-dispersion wavelengths around the wavelengths of $1980(\mathrm{~nm})$ and $2800(\mathrm{~nm})$. 


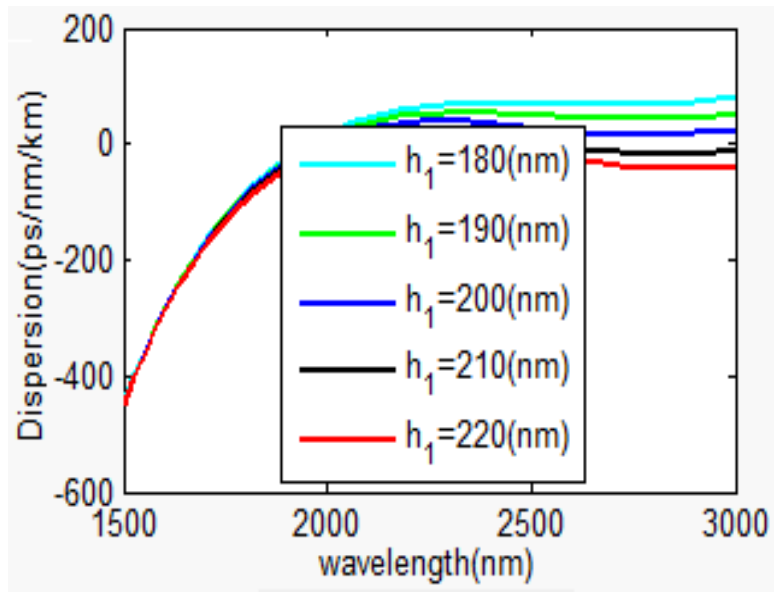

(a)

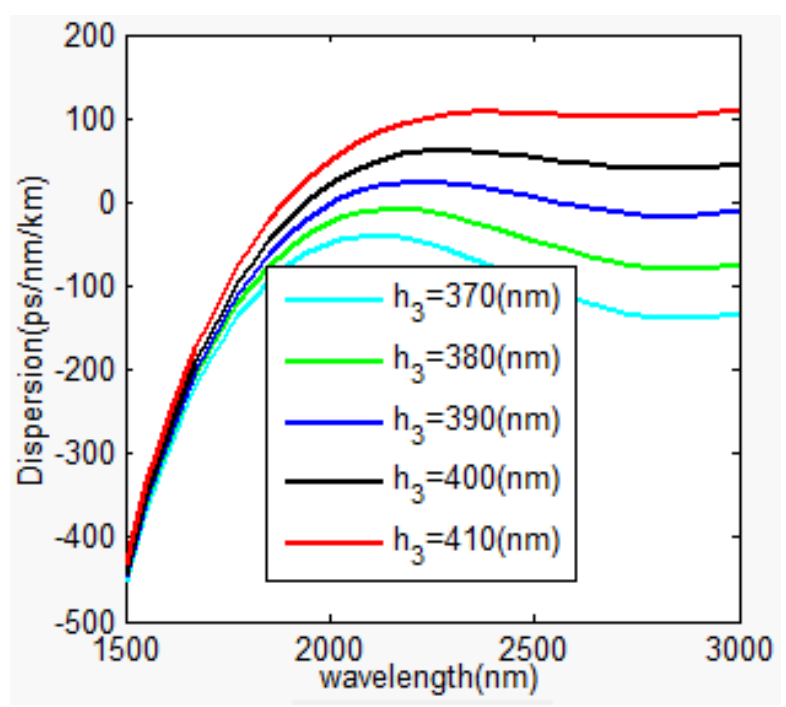

I

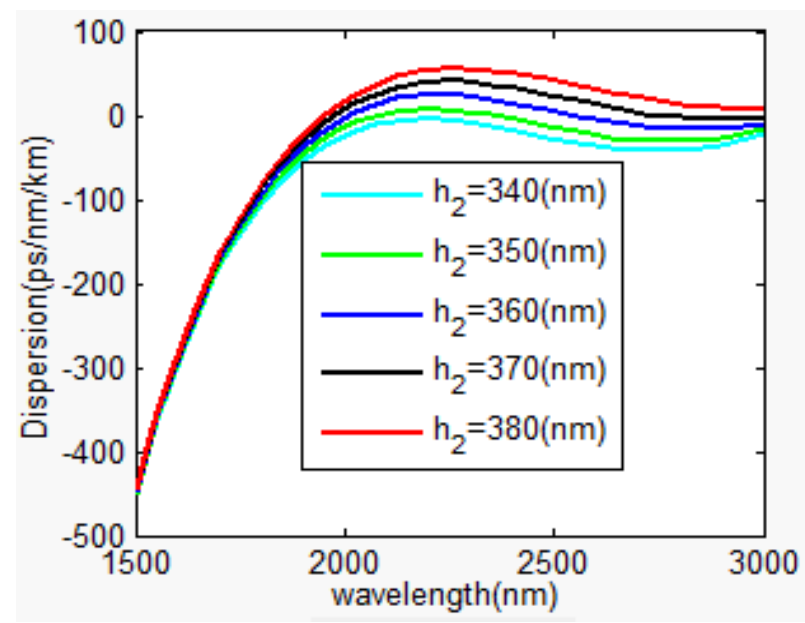

(b)

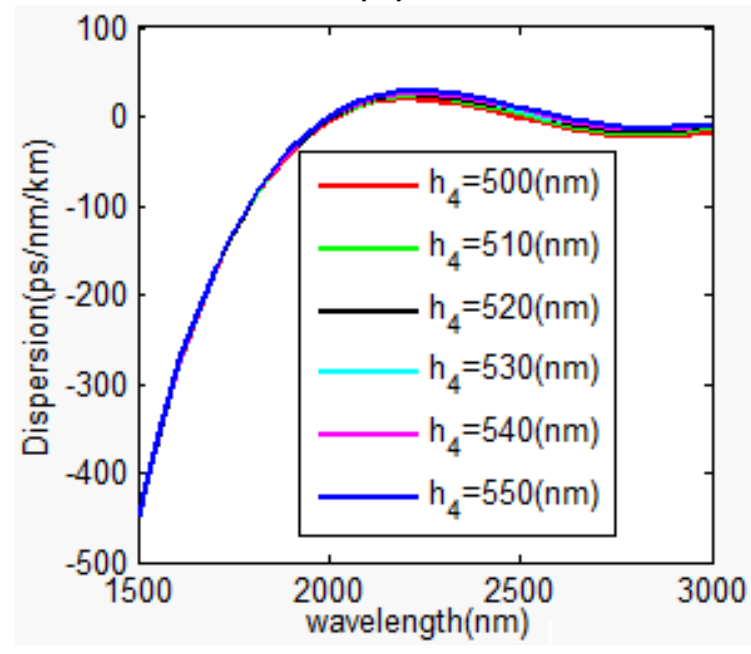

(d)

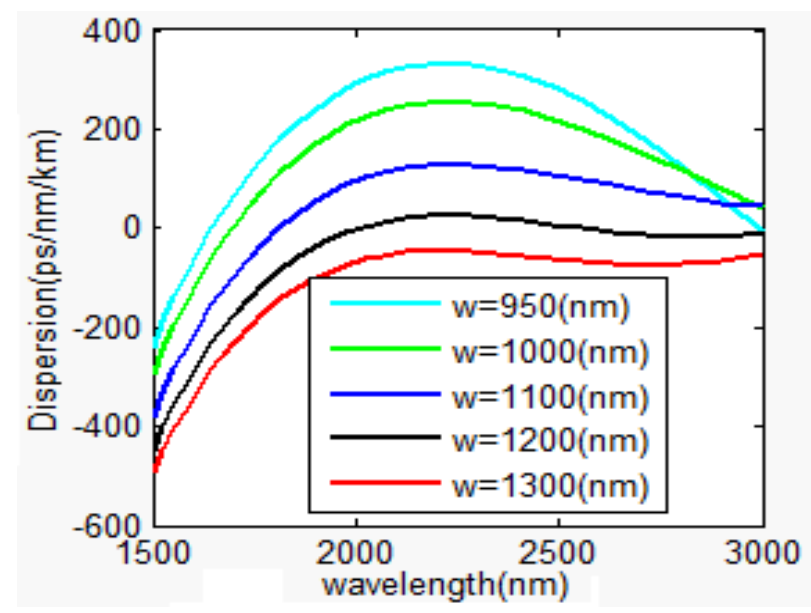

(e)

Fig2: Dispersion profiles of structure 2, (a) Dispersion changes with respect to h1, (b) Effect of $h_{2}$ varying on dispersion profile, (c) Dispersion changes with respect to h3, (d) h4 and (e) w. 
Table 1

Design parameters of the proposed sensor.

\begin{tabular}{lccccc}
\hline Parameter & $\mathrm{h}_{1}$ & $\mathrm{~h}_{2}$ & $\mathrm{~h}_{3}$ & $\mathrm{~h}_{4}$ & $\mathrm{~W}$ \\
Value & $200(\mathrm{~nm})$ & $360(\mathrm{~nm})$ & $390(\mathrm{~nm})$ & $540(\mathrm{~nm})$ & $1100(\mathrm{~nm})$
\end{tabular}

By numerically solving Equation 1 by the split-step Fourier method, one can observe the spectrum of generated supercontinuum spectra in the structure and properties of the FCG. To investigate the supercontinuum spectrum, an input pulse with a power of $800(\mathrm{w})$ and a pump pulse width of 50 (fs) is ainjected to the input of waveguide 1 . The value of the effective mode area in this structure is $0.33 \mu \mathrm{m}^{2}$ which, according to the nonlinear coefficient of silicon $n_{2}=12 \times 10^{-14} \mathrm{~cm}^{2} \mathrm{w}^{-1}$ at about of $1900 \mathrm{~nm}$ (Bristow et al. 2007), the value of the nonlinear coefficient is equal to $\gamma=115.93 \mathrm{~m}^{-1} \mathrm{w}^{-1}$ is obtained. The supercontinuum spectrum and the corresponding spectrum evolution are shown in Fig3. This spectrum is prepared from wavelengths of $1100(\mathrm{~nm})$ to more than $4000(\mathrm{~nm})$.

For use in optical communications and the production of supercontinuum based frequency combs, the output spectrum is also simulated by numerically solving the Schrödinger equation at a pumping wavelength of $1550(\mathrm{~nm})$ in the normal dispersion region. To create effective interactions between dispersion and nonlinear effects, the waveguide length is considered to be $8(\mathrm{~mm})$ in this case. The maximum input power $1(\mathrm{KW})$ with a pulse width of $100(\mathrm{fs})$ is considered, where Equation 1 can be considered simpler (Liu et al. 2007; Muslu G. and Erbay 2005).

Fig4 shows the spectrum corresponding to the observed ultra broadband comb obtained via the structure1. There are several sharp peaks in the spectrum, which 
indicates the ability to frequency combs generation in this structure (Goji et al. 2021; Enomoto et al. 2016; Yoshii et al. 2019).

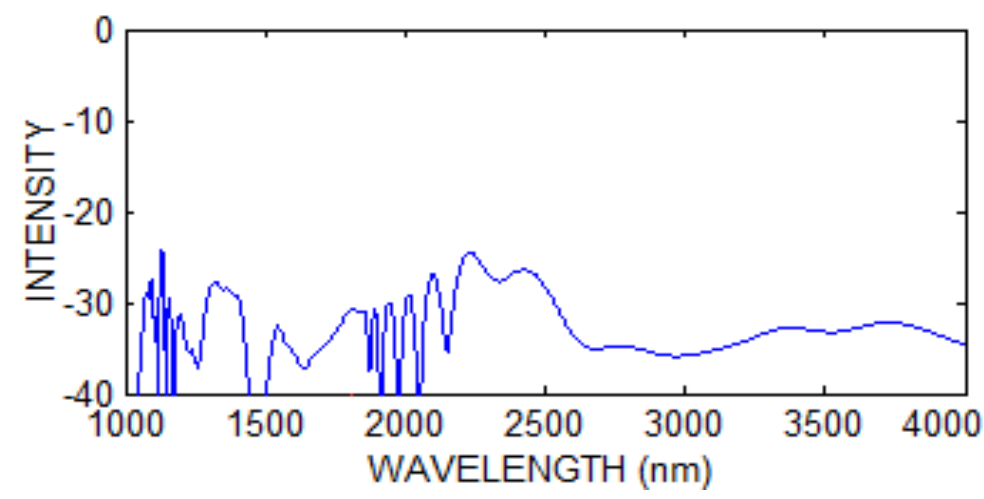

(a)

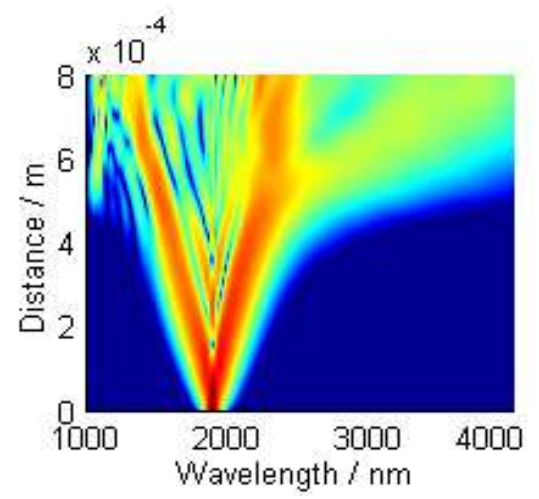

(b)

Fig3. supercontinuum spectrum generated in structure 1, (a) supercontinuum spectrum with a pumping wavelength of 1890 (nm), (b) spectrum evolution.

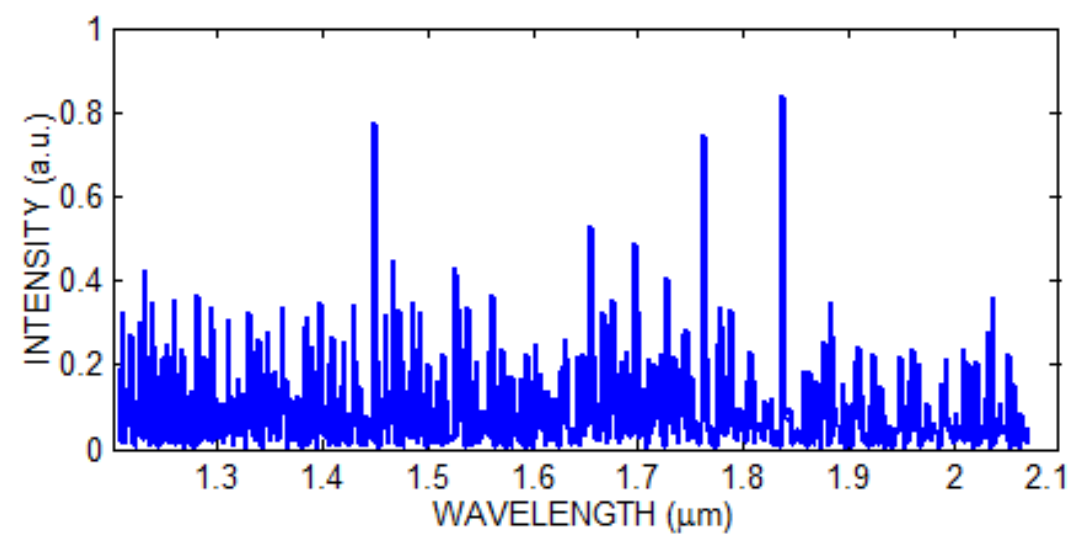

Fig4: Frequency comb spectrum generated in structure 1 with pumping wavelength of $1550(\mathrm{~nm})$.

To generate supercontinuum spectrum in structure 2 , an input pulse with a peak power of $800(\mathrm{w})$ and a pulse width of $50(\mathrm{fs})$ at a wavelength of $1890(\mathrm{~nm})$ is injected to a $0.8 \mathrm{~mm}$ waveguide input. The effective area of mode in this structure is $0.4 \mu m^{2}$, and the nonlinear coefficient is calculated like the first structure, the 
value of which is $\gamma=99.68 m^{-1} w^{-1}$. Fig. 5(a) and (b) show the mid-IR spectral broadening and pulse evolution in proposed waveguide2.

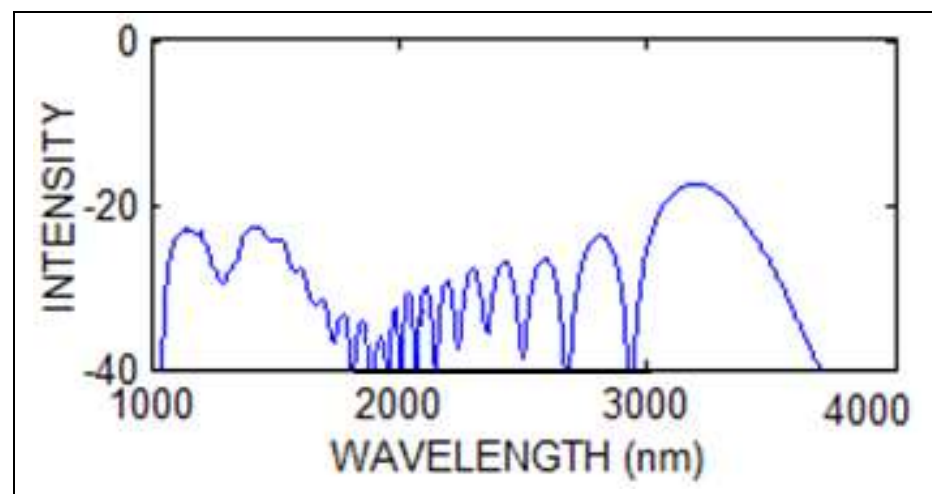

(a)

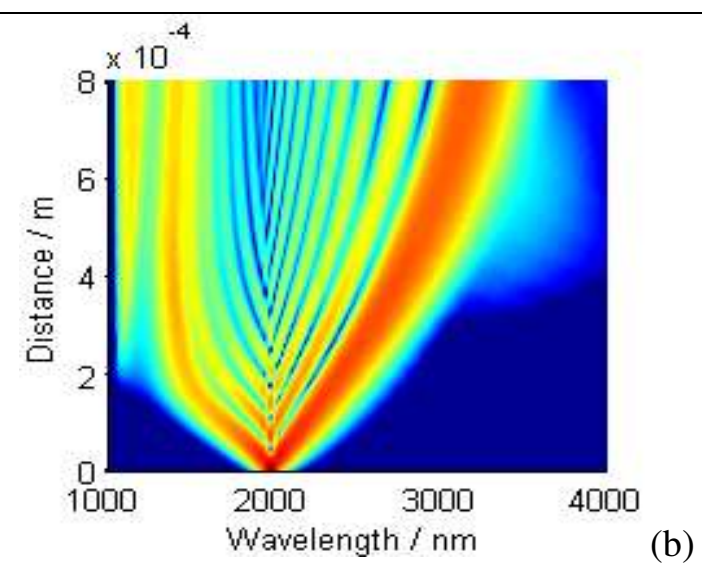

Fig5. supercontinuum spectrum generated in structure 2, (a) supercontinuum spectrum with a pumping wavelength of $1890(\mathrm{~nm})$, (b) pulse evolution.

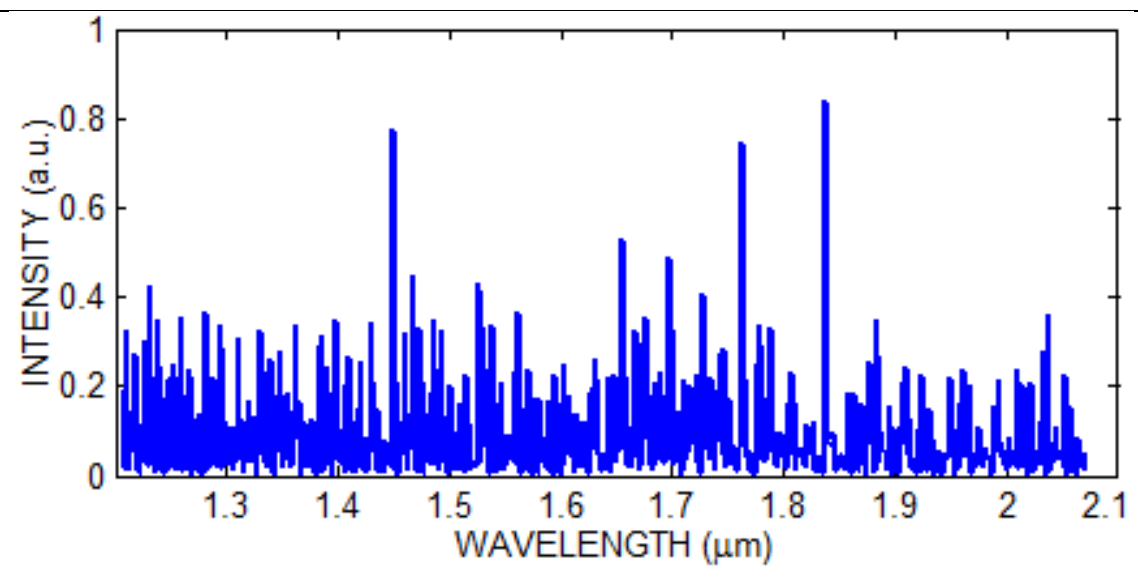

Fig6: Frequency comb spectrum generated in structure 2 with pumping wavelength of 1550 (nm).

As can be seen in this figure, the output spectrum is normal and flat at wavelengths between $1000(\mathrm{~nm})-1800(\mathrm{~nm})$ and can be seen blue and red shifted solitons that cause spectral broadening from $1100(\mathrm{~nm})$ to $3800(\mathrm{~nm})$. 
Fig 6 also shows the comb properties of waveguide 2 by numerically solving Eq. 1 and injecting an input pulse at the wavelength of $1550(\mathrm{~nm})$. The comb properties of the output spectrum of waveguide 2 can be observed in this figure like fig 4 .

By comparing the two output spectra related to frequency combs, it is observed that the structure 2 is more suitable for generation of flat frequency combs in optical communication regime.

\section{Conclusion}

In this paper, by dispersion engineering and investigating the effect of dimensions on the dispersion profile, two silicon waveguides are proposed to generation of supercontinuum spectrum and frequency combs based on supercontinuum spectrum. For SCG, the pump pulse with peak power 800 (w) and pulse duration of 50 (fs) is injected to the input of both waveguides with a length of $0.8(\mathrm{~mm})$. The first zero dispersion wavelengths located near the $1980(\mathrm{~nm})$ and $1890(\mathrm{~nm})$ in the waveguides 1 and 2 respectively. However, to investigate the frequency comb generation characteristics of the waveguides, an input pulse with a pulse duration of 100 (fs) is applied in the normal dispersion region of both waveguides at the wavelength of $1550(\mathrm{~nm})$. The supercontinuum spectrum in waveguide 1 and 2 has been achieved from the wavelength of $1100-4000(\mathrm{~nm})$, and $1200-3800(\mathrm{~nm})$ respectively. The generated optical frequency combs also have a wide bandwidth and can cover the optical communication area. The proposed structures can be used in optically integrated circuits, optical communications and spectroscopy.

\section{Funding}

The authors did not receive support from any organization for the submitted work. 


\section{References}

Aggarwal .I.D., Sanghera .J.S.: Development and applications of chalcogenide glass optical fibers at NRL. J. Optoelectron. Adv. Mater. 4, 665-678 (2002). 10.1016/S0022-3093(97)00051-3

Agrawal G. P., Nonlinear Fiber Optics, 5th ed. Elsevier Academic Press, Oxford, UK, (2013).

Alizadeh M. R., Seifouri M.: Investigation of Highly Broadb and Supercontinuum Generation in a Suspended $\mathrm{As}_{2} \mathrm{Se}_{3}$ Based Ridge Waveguide. J. of Opto.. Nano., 5(4), (2020). 20.1001.1.24237361.2020.5.4.1.2

Alizadeh M.R, Seifouri M.: Dispersion engineering of highly nonlinear rib waveguide for mid-infrared super continuum generation. Optik 140, 233-238 (2017). 10.1016/j.ijleo.2017.04.056

Alizadeh M. R., Seifouri M.: Design and Analysis of a Dispersion-engineered and Highly Nonlinear Rib Waveguide forGeneration of Broadband Supercontinuum Spectra. Freq. (2019). https://doi.org/10.1515/freq-2019-0098

Amirhassan. S., L. Pawel, O. Yoshitomo, H. Gary, P. Nathalie, G. Alexander, and L. Marko.: Supercontinuum generation in angle-etched diamond waveguides. Opt. Lett. 44(16), 4056-4059 (2019).

https://doi.org/10.1364/OL.44.004056

Baohua Luo ; Yingying Wang ; Shixun Dai ; Ya'nan Sun ; Peiqing Zhang ; Xunsi Wang ; Feifei Chen.: Midinfrared Supercontinuum Generation in $\mathrm{As}_{2} \mathrm{Se}_{3}-\mathrm{As}_{2} \mathrm{~S}_{3}$ Chalcogenide Glass Fiber With High NA. Journal of Lightwave Technology, 35(12), 2464 - 2469(2017). 10.1109/JLT.2016.2623639

Bristow Alan D., Nir Rotenberg, and Henry M. van Driel.: Two-photon absorption and Kerr coefficients of silicon for 850-2200 nm. Appl. Phys. Lett. 90, 191104 (2007). https://doi.org/10.1063/1.2737359

Chang. L., A. Boes, X. Guo, D. T. Spencer, M. Kennedy, and J. E. Bowers.: Heterogeneously Integrated GaAs Waveguides on Insulator for Efficient Frequency Conversion. Laser Photonics Rev. 12, 1800149 (2018). https://doi.org/10.1002/lpor.201800149

Company V.T., Weiner A.M.: Optical frequency comb technology for ultrabroadband radio-frequency photonics. Laser Photonics, 8(3) 368-393(2014). 10.1002/Ipor.201300126

Da. F. Ros, M. Pu, L. Ottaviano, H. Hu, E. Semenova, M. Galili, K. Yvind, and L. K. Oxenlowe.: Phase-sensitive four-wave mixing in AlGaAs-on-insulator nano-waveguides. IEEE Photon.s Con. (IPC), 505-506(2016). 10.1109/IPCon.2016.7831202

Dai. Shixun, Yingying Wang, Xuefeng Peng, Peiqing Zhang, Xunsi Wang and Yinsheng Xu. A.: Review of MidInfrared Supercontinuum Generation in Chalcogenide Glass Fibers. Appl.Sci. 8,707 (2018).

$\underline{10.3390 / a p p 8050707}$

Deng Yi, Chunjiang Wu, Yu Liu, Suchun Feng.: Dual-pumped flat optical frequency comb based on normal dispersion AlGaAs on insulator waveguide: Numerical investigation. Optics Communications, 502, 127415 (2022). https://doi.org/10.1016/i.optcom.2021.127415

Dudley. J.M., G. Genty, S. Coen.: Supercontinuum generation in photonic crystal fiber. Rev. Mod. Phys. 78,11351184 (2006). https://doi.org/10.1103/RevModPhys.78.1135

Dudley.J.M., Taylor.J.R.: Ten years of nonlinear optics in photonic crystal fiber. Nat. Photon. 3, 85-90 (2009). https://doi.org/10.1038/nphoton.2008.285 
Dupont, C. Petersen, J. Thogersen, C. Agger, O. Bang, and S. R. Keiding.: IR microscopy utilizing intese supercontinuum light source. Opt. Express 20(5), 4887-4892 (2012). https://doi.org/10.1364/OE.20.004887

Eggleton. B.J., B. Luther-Davies, K. Richardson.: Chalcogenide photonics. Nat. Photon. 5, 141-148 (2011). https://doi.org/10.1038/nphoton.2011.309

Enomoto, K., Hizawa, N., Suzuki, T., Kobayashi, K., Moriwaki, Y.: Comparison of resonance frequencies of major atomic lines in 398-423 nm. Appl. Phys. B 122(5), (2016). 10.1007/s00340-016-6400-5

Feifle. J. P, V. Brasch, M. Lauermann, Y. Yu, D. Wegner, T. Herr, K. Hartinger, et al.: Coherent terabit communications with microresonator Kerr frequency combs. Nat. Photon. 8 375-380 (2014). https://doi.org/10.1038/nphoton.2014.57

Frigg. A, A. Boes, G. Ren, I. Abdo, D. Choi, S. Gees, and A. Mitchell.: Low loss CMOS-compatible silicon nitride photonics utilizing reactive sputtered thin films. Opt. Express 27, 37795 (2019).

https://doi.org/10.1364/OE.380758

Ghanbari Ashkan, Alireza Kashaninia, Ali Sadr, Hamed Saghaei.: Supercontinuum generation with femtosecond optical pulse compression in silicon photonic crystal fibers at $2500 \mathrm{~nm}$. Optical and Quantum Electronics, 50:411(2018). https://doi.org/10.1007/s11082-018-1651-5

Goji Yuma, Chaoyun Chen, Kohei Ikeda, Kazumichi Yoshii, Feng-Lei Hong.: Towards generation of optical frequency comb in the short-wavelength visible region using periodically poled lithium niobate waveguides. Results in Optics, 2, 100035 (2021). https://doi.org/10.1016/i.rio.2020.100035

Gong. Z., A. Bruch, M. Shen, X. Guo, H. Jung, L. Fan, X. Liu, L. Zhang, J.Wang, J. Li, J. Yan, and H. Tong.: Highfidelity cavity soliton generation in crystalline AlN micro-ring resonators. Opt. Lett. 43(18), 4366-4369 (2018). https://doi.org/10.1364/OL.43.004366

Guo Yuhao, Jafari Zeinab, Lijuan Xu, Changjing Bao, Peicheng Liao, Guifang Li, Anuradha M. Agarwal, Lionel C. Kimerling, Jurgen Michel, Alan E. Willner, and Lin Zhang.: Ultra-flat dispersion in an integrated waveguide with five and six zero-dispersion wavelengths for mid-infrared photonics. Photonics Research 7(11), (2019). https://doi.org/10.1364/PRJ.7.001279

Hu .H., F. Ros, M. Pu, F. Ye, K. Ingerslev, E. Silva, Y. Amma, Y. Sasaki, T. Mizuno, T. Morioka, and L. Oxenlowe.: Single-source chip-based frequency comb enabling extreme parallel data transmission. Nat. Photon. 12, 469-473 (2018). https://doi.org/10.1038/s41566-018-0205-5

Hu . H., Oxenløwe .L.K., "Chip-based optical frequency combs for high-capacity optical communications", Nanophotonics 10(5), (2021). doi.org/10.1515/nanoph-2020-0561

Husakou. A. V. and J. Herrmann.: Supercontinuum generation of higher-order solitons by fission in photonic crystal fibers. Phys. Rev. Lett. 27, 203901 (2001). https://doi.org/10.1103/PhysRevLett.87.203901

Karami. Rahim, Mahmood Seifouri, Saeed Olyaee, Mohammad Chitsazian1 and Mohammad Reza Alizadeh, "Numerical analysis of a circular chalcogenide/silica hybrid nanostructured photonic crystal fiber for the purpose of dispersion compensation" Int. J. Numer, 30: e2184 (2017). https://doi.org/10.1002/jnm.2184

Karpisz Tomasz, Bartlomiej Salski, Anna Szumska, Mariusz Klimczak, Ryszard Buczynski. "FDTD analysis of modal dispersive properties of nonlinearphotonic crystal fibers", Opt. Quant. Electron. 47, 99-106(2015). 
https://doi.org/10.1007/s11082-014-9987-y

Ke, K.; Xia, C.; Islam, M.N.;Welsh, M.J.; Freeman, M.J.: Mid-infrared absorption spectroscopy and differential damage in vitro between lipids and proteins by an all-fiber-integrated supercontinuum laser. Opt. Express, 17, 12627-12640 (2009). 10.1364/OE.17.012627

Kippenberg T.J., A.L. Gaeta, M. Lipson, M.L. Gorodetsky.: Dissipative Kerr solitons in optical microresonators. Science 361(6402), 8083(2018). 10.1126/science.aan8083

Li. M., L. Zhang, and D. X. Dai.: Hybrid silicon nonlinear photonics Photonics. Res. 6(5), B13-B22 (2018). https://doi.org/10.1364/PRJ.6.000B13

Liu G., Z. Lu, J. Liu, Y. Mao, M. Vachon, C. Song, P. Barrios, et al.: Passively mode-locked quantum dash laser with an aggregate 5.376 Tbit/s PAM-4 transmission capacity. Opt. Express 28(4), 4587-4593(2020). https://doi.org/10.1364/OE.386266

Liu Y., J. E. Sharping, and M. T. Cicerone.: Numerical simulation of supercontinuum generation in $\mathrm{GeO}_{2}$ doped fiber. Proc. of SPIE 6698 (2007). https://doi.org/10.1117/12.738606

Ma. P., D. Y. Choi, Y. Yu, X. Gai, Z. Yang, S. Debbarma, S. Madden, and B. Luther-Davies.: Low-loss chalcogenide waveguides for chemical sensing in the mid-infrared. Opt. Express 21(24), 29927-29937 (2013).

McCarthy J., H. Bookey, S. Beecher, R. Lamb, I. Elder, and A. K. Kar.: Spectrally tailored mid-infrared supercontinuum generation in aburied waveguide spanning $1750 \mathrm{~nm}$ to $5000 \mathrm{~nm}$ for atmospherictransmission. Appl. Phys. Lett. 103(15), 151103 (2013). https://doi.org/10.1063/1.4824358

Ming C., Qing Y., Tiansong L., C. Mingsong, H. Ning, "New high negative dispersion photonic crystal fiber", New Optik 121, 867-871 (2010). https://doi.org/10.1016/j.ijleo.2008.09.039

Møller. U.,Y.Yu,I.Kubat,C.R.Petersen,X.Gai,L.Brilland,D.Méchin,C.Caillaud,J.Troles,B.Luther Davies,and O.Bang.: Multi-milliwattmid-infrared supercontinuum generation in a suspended core chalcogenide fiber. Opt.Exp.23,3282(2015). https://doi.org/10.1364/OE.23.003282

Moss. D. J., R. Morandotti, and M. Lipson.: New CMOS-compatible platforms based on silicon nitride and Hydex for nonlinear optics. Nat. Photonics 7, 597 (2013). https://doi.org/10.1038/nphoton.2013.183

Muslu G. and Erbay H.: Higher-order split-step Fourier schemes for the generalized nonlinear Schroedinger equation. Mathematics and Computers in Simulation 67, 581 (2005).

https://doi.org/10.1016/i.matcom.2004.08.002

Myslivets E., Kuo B.P.P., Alic N., Radic S., "Generation of wideband frequency combs by continuous-wave seeding of multistage mixers with synthesized dispersion”, Opt. Express 20, 3331-3344 (2012).

https://doi.org/10.1364/OE.20.003331

Obrzud E., M. Rainer, A. Harutyunyan, B. Chazelas, M. Cecconi, A. Ghedina, E. Molinari, et al.: Broadband nearinfrared astronomical spectrometer calibration and on-sky validation with an electro-optic laser frequency comb. Opt. Express 26(26), 34830-34841 (2018). https://doi.org/10.1364/OE.26.034830

Obrzud. E., M. Rainer, A. Harutyunyan, M.H. Anderson, J. Liu, M. Geiselmann, B. Chazelas, et al.: A microphotonic astrocomb. Nat. Photon. 13, 31-35 (2019). https://doi.org/10.1038/s41566-018-0309-y 
Oxenlowe. L. K., Hua Ji, M. Galili, Minhao Pu, Hao Hu, H. C. H. Mulvad, K. Yvind, J. M. Hvam, A. T. Clausen, and P. Jeppesen.: Silicon Photonics for Signal Processing of Tbit/s Serial Data Signals. IEEE J. Sel. Top. Quantum Electron., 18(2), 996-1005 (2012). 10.1109/JSTQE.2011.2140093

Paul M. Walker, Charles E. Whittaker, Dmitry V. Skryabin, Emiliano Cancellieri, Ben Royall, Maksym Sich, Ian Farrer, David A. Ritchie, Maurice S. Skolnick and Dmitry N. Krizhanovskii. "Spatiotemporal continuum generation in polariton waveguides" Science \& Applications 8(6) 1-11( 2019). https://doi.org/10.1038/s41377-019-0120$\underline{7}$ $\mathrm{Pu}$.Minhao, Luisa Ottaviano, Elizaveta Semenova, Francesco Da Ros, Hao Hu, Ayman Nasar Kamel, Yi Zheng, Erik Stassen, Michael Galili, Leif Katsuo Oxenløwe, and Kresten Yvind.: An ultra-efficient nonlinear planar integrated platform for optical signal processing and generation. Asia Communications and Photonics Conference (ACP), OSA (2017). https://doi.org/10.1364/ACPC.2017.S4J.6

Pu. M., L. Ottaviano, E. Semenova, and K. Yvind.: Efficient frequency comb generation in AlGaAs-on-insulator. Optica 3(8), 823-826 (2016). https://doi.org/10.1364/OPTICA.3.000823

Qin. Jun, Haowen Shu, Lin Chang, Weiqiang Xie, Yuansheng Tao, Ming Jin, Xingjun Wang, AND John E. Bowers.: On-chip high-efficiency wavelength multicasting of PAM3/PAM4 signals using low-loss AlGaAs-oninsulator nanowaveguides. Optics Letters. 45(16), 4539-4542 (2020).

https://doi.org/10.1364/OL.398777

Safioui, J. et al.: Supercontinuum generation in hydrogenated amorphous silicon waveguides at telecommunication wavelengths. Opt. Express 22, 3089-3097 (2014). https://doi.org/10.1364/OE.22.003089

Seifouri. M. and Alizadeh M. R.: Supercontinuum Generation in a Highly Nonlinear Chalcogenide/ $\mathrm{MgF}_{2} \mathrm{Hybrid}$ Photonic Crystal Fiber. IJOP, 12(1), (2018). http://ijop.ir/article-1-277-en.html

Takushima Y., F. Futami, K. Kikuchi.: Generation of over 140-nm-wide supercontinuum from a normal dispersion fiber by using a mode-locked semiconductor laser source. IEEE Photonics Technol. Lett. 10, 1560-1562 (1998). $\underline{10.1109 / 68.726749}$

Tan. D. T. H., K. J. A. Ooi, and D. K. T. Ng.: Nonlinear optics on silicon-rich nitride - a high nonlinear figure of merit CMOS platform. Photonics Res. 6(5), B50 (2018). https://doi.org/10.1364/PRJ.6.000B50

Willner. A. E, A. Fallahpour, F. Alishahi, Y. Cao, A. Almaiman, P. Liao, K. Zou, A. N. Willner, and M. Tur, J.: AllOptical Signal Processing Techniques for Flexible Networks. Lightwave Technol. 37, 21 (2019).

$\underline{10.1109 / J L T .2018 .2873245}$

Xue. X., X. Zheng, B. Zhou, A.M. Weiner.: Microresonator frequency combs for integrated microwave photonics. IEEE Photo. Tech. Lett. 30 (21) 1814-1817 (2018). 10.1109/LPT.2018.2875945

Yoon, Oh. D. et al.: Coherent ultra-violet to near-infrared generation in silica ridge waveguides. Nat. Commun. 8, 13922 (2017). https://doi.org/10.1038/ncomms13922

Yoshii, K., Nomura, J., Taguchi, K., Hisai, Y., Hong, F.-L.: Optical Frequency Metrology Study on Nonlinear Processes in a Waveguide Device for Ultrabroadband Comb Generation. Phys. Rev. Appl. 11, 054031(2019). https://doi.org/10.1103/PhysRevApplied.11.054031

Yu.,X.Gai,P.Ma,D.Y.Choi,Z.Y.Yang,R.P.Wang,S.Debbarma,S.J. Madden, and B. Luther-Davies.: A broadband, quasi-continuous, mid-infrared supercontinuum generated in a chalcogenide glass waveguide. Laser Photon. Rev. 8(5), 792-798 (2014). https://doi.org/10.1002/lpor.201400034 
Zhang M., B. Buscaino, C. Wang, A.S. Ansari, C. Reimer, R. Zhu, J.M. Kahn, et al.: Broadband electro-optic frequency comb generation in a lithium niobate microring resonator. Nature 568 373-377(2019).

https://doi.org/10.1038/s41586-019-1008-7

Zhang Y., C. Qian, D. Li, S. Zhang, J. Zhao.: Long-term stabilization of an actively mode-locked Er-doped fiberring laser via dynamic intracavity loss feedback. J. Lightwave Technol. 34, 3959-3965(2016).

10.1109/JLT.2016.2597294

Zho Z., Brown T., "Full-vectorial finite-difference analysis of microstructure optical fibers", Opt. Express 10 (85) 3-64(2002). https://doi.org/10.1364/OE.10.000853 